\title{
Author Index Vol. 27, 1995
}

Abalain, M.-L. 322 Alio,J.L. 136 Amemiya, T. 249 Angulo, S. 37 Araki, H. 208 Artola,A. 136 Ayala,M.J. 136 Azzam, N. 197

Baerveldt,G. 163 Bartov, E. 23 Bazan,N.G. 153 Bellot,J. 136 Bergmann, L. 67 Bhutto, LA. 249 Bonhomme, B. 153 Braquet,P. 153 Buzzonetti, L. 168

Campbell, RJ. 42 Carreras, F.J. 53 Chang, S.-W. 74 Chen,H.-B. 158,286 Chen,M.-S. 74 Cheng, H.-M. 12 Cluzel,J. 153 Colin, J. 322 Colotto,A. 168 Coppè,A. 168 Cuevas-Sanchez, P. 317

DeLuca,L.A. 168 Desatnik, H. 23 Devamanoharan, P.S.

18 Doly,M. 102,153 Doughty, MJ. 80 Dovrat,A. 197 Droy-Lefaix, M.T. 102

Fagerholm, P. 317 Falsini,B. 168 Fujii,Y. 158 Fujisawa, K. 89 Fukumaki,Y. 310

Gershon,D. 197 Giudiceandrea, A. 168 Glonek,T. 277

Gonzalez, O. 37 Grande, J.P. 42 Greiner,J.V. 277 Guerra, A. 37

Hamamoto, M. 310 Hartmann, Ch. 67 Hashizume, N. 129,335 Hata, Y. 48 Hayasaka, S. 347

Hayashi, A. 262 Hilgers, R.D. 67 Hiraki,S. 347 Hirose,T. 234 Hockwin, O. 178 Hollander, H.

296 Honda, Y. 118 Hsu,H.-C. 74 Hu,F.-R. 74

Igarashi, H. 12 Inomata,H. 48,208,

310,330 Ishibashi,T. 48,208,

330 Itabashi, R. 234 Iwatsuru, M. 350

Jauch, A. 322 Johnson, J.A. 42

Kamei, M. 262 Kato,K. 227 Katsumi,O. 234 Kawano,Y.-I. 310 Kawasaki, K. 57,110 Khalil,A. 48,330 Kim,Y.Y. 243 Kobata,S. 129 Kobayashi,T. 219 Koenig, J.L. 268 Kojima,M. 178,189

Koliopoulos, I. 67 Komata, M. 110 Krishna, R. 163 Kumano,Y. 310 Kumar, R. 42

Leahy, CD. 277 Lee,J.W. 350 Lee,T.S. 243

Majima, K 202, 356 Makarov, F. 296 Malet,F. 322 Malik, A. 189 Manabe, J.-i. 310 Matsumura, M. 118 Mehta, M.C. 234 Mermoud, A. 163 Minagawa, H. 310 Minckler, D.S. 163 Mulet,M.E. 136 Murata,T. 48,330

Nagai,Y. 118 Naka,H. 262 Naveh,N. 23 Nguyen, KT. 80 Nishigori, H. 350 Nishimoto, K. 1 Numata,K. 347 Nussbaum, A. 23 Nussenblatt, R. 366

Ogino,N. 118 Oguni, M. 227 Ohnishi,Y. 335 Ohno,S. 158,219,286 Okada,M. 118 Okada,Y. 129,335 Ooshima,A. 129,335 Ou,B. 158,286

Pastor, J.C. 37 Pharmakakis, N. 67 Porcel, D. 53

Roche, P.C. 42 Ruiz,J.M. 136

Saavedra, J.A. 37 Saika,S. 129,335 Sakagami, K. 262 Sakai,H. 57 Sakata, H. 32 Salgarello, T. 168

Sasabe, T. 262 Sasaki, K. 1,89, 178, 189 Schapira,D. 197 Scharf,J. 197 Seko,Y. 144 Setogawa, T. 227 Setten, G. van 317 Shaw,E.M. 268 Shinohara, H. 227 Shirao, Y. 57,110 Shirasawa, E. 57 Shui,Y.B. 178 Silbermann, M. 197 Stefani,F.H. 296 Stone, J. 296 Sugai,S. 208 Szabo,M.E. 102 Tamura, H. 227 Tanaka, K 12 Tanaka, O. 227 Tanaka, S.-i. 335 Tanaka, Y. 144 Tani,Y. 57 Tano,Y. 262 Tawara, A. 208 Tokoro,T. 144 Tsukahara,S. 158,219, 
286 Tsumura, K. 32

Ueda,H. 219

Varma,S.D. 18 Vrensen, G.F.J.M. 187

Wajima,R. 234 Watanabe, M. 32,110 Weale,R.A. 214 Weber, KH. 188 Williams, T.R. 268

Yamabayashi, S. 158,286 Yamanaka,O. 129,335 Yano,H. 347 Yoshida,A. 12 Yoshikawa, H. 48, 330

Zrenner, E. 64

368 